\title{
Current Developments on the zbMATH Interface
}

Octavio Paniagua Taboada (FIZ Karlsruhe, Berlin, Germany)

Like every year, zbMATH's staff of editors and developers has introduced new ideas and useful tools to enrich our database and provide the users with an easier and comfortable experience while using zbMATH. These developments have considered the great feedback of the mathematical community and other users of zbMATH, e.g., librarians.

\section{New search options for authors and serials}

Perhaps it has happened to you that looking for the articles of a certain author you find the work of other authors having the same name(s). Any additional information, like a middle name, could be really helpful in finding the author you are looking for. We have introduced some new fields (commands) that you can use in the author tab using one-line search. These fields are "In" (last name) and "fn" (first name). Suppose that you are interested in an author called Wei Li. Then you can type the following commands in the author tab (one-line search): In:Li fn:Wei. You will find 72 author profiles that correspond to that name ${ }^{1}$ (in this case, $\mathrm{Li}$ and Wei could be both first name and surname). If we now invert the order fn:Li In:Wei we find 14 author profiles. Compare this with the 122 results that we find if we just search for Wei $\mathrm{Li}$ in the author tab. In these cases or when the authors are exact homonyms, any additional information, such as mathematical area, or the profile in external databases like the Mathematics Genealogy Project, allows us to correctly distinguish them. If you already know exactly which author you are looking for, then it is really useful to use her/his personal author identifier "ai". You can also do a structured search in the author tab. This structured search looks like this:

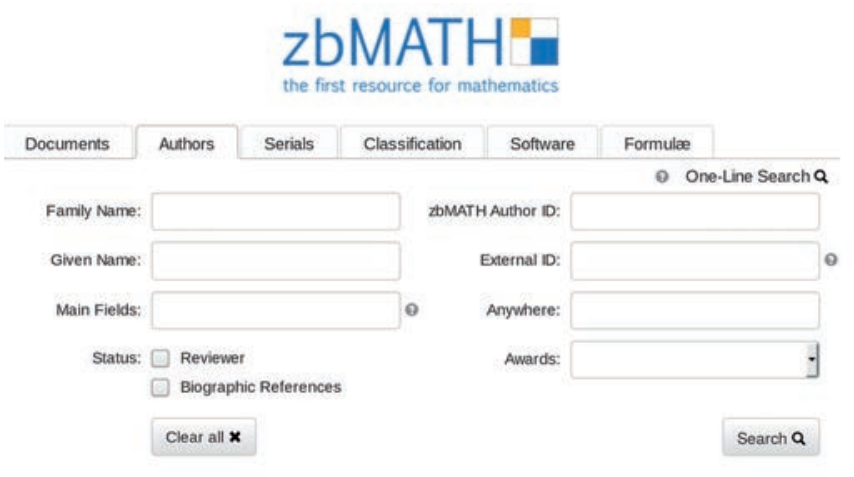

1 Based on the current index on February 3rd 2019.
Notice that there is more information that you can provide about the author you are looking for, e.g., awards, main fields, zbMATH author ID, or other external IDs (such as MGP and ORCID).

Likewise, we have implemented similar improvements for serials. In the serials tab (formerly called "journals") you can use the one-line search and an enhanced structured search. In the one-line search you can use the following fields: "jt" (meaning journal or serial title), "pu" (publisher), "sn" (ISSN code), "tp" (type of publication), and the field "st" allows you to find, for instance, open access journals (st: vo). There is also a new structured search at the serials tab, where you have additional options like language, time period, country, main fields, etc.

Additionally, you will find some small question marks (this is also the case for the author structured search), which explain the possibilities that you have or provide you with some examples. The structured search for series has this appearance:

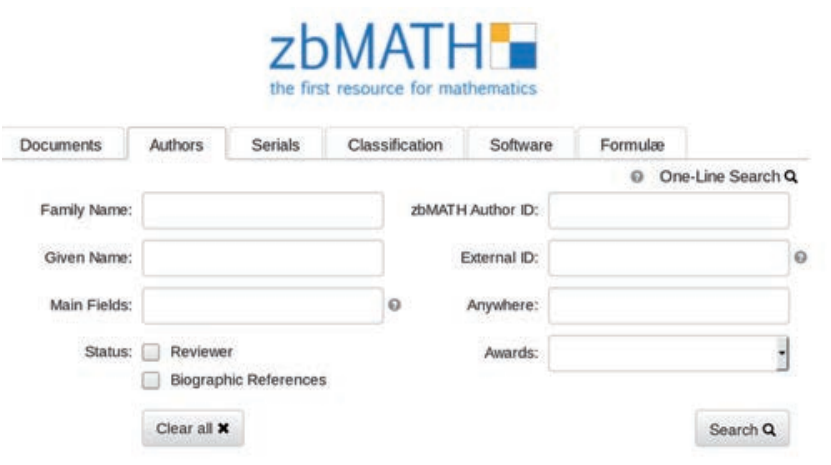

As a final remark, you will notice that the documents structured search also has a few changes.

\section{New filters and facets at the zbMATH interface}

zbMATH now has some new filters that are displayed after you make a query. Among these filters you will find Document Type (serial articles, collections articles, books), Reviewing State (reviewed, title only, pending, scanned review), Biographic Reference, Software, and Volume (this filter appears when all results of your search belong to the same serials). Furthermore, when you load a series profile, you find the facet "Latest Issues", where it is possible to check the content of the latest volumes. In the figure below you can see a current series profile: 
Annals of Mathematics. Second Series

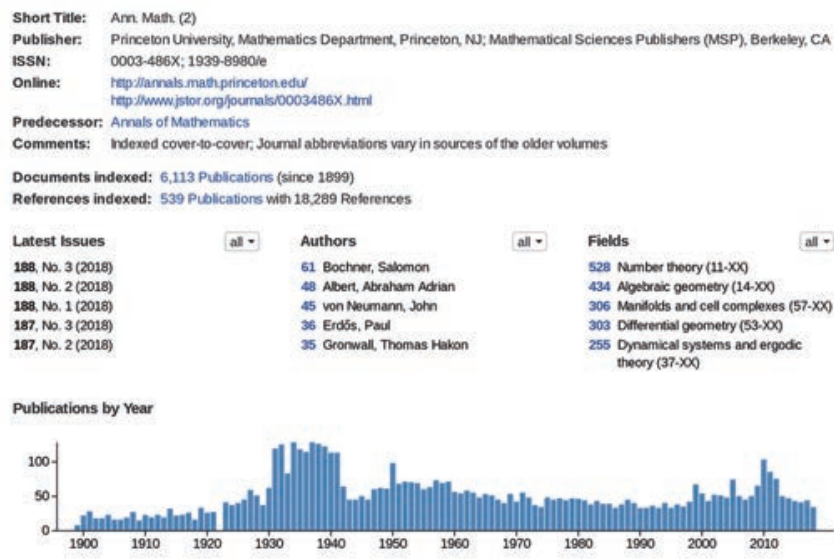

Citations contained in zbMATH

Loading cetation profile.

In addition, you will notice the new look of the results page when you perform a serials query.

\section{zbMATH on Twitter}

Social networks have proved capable of reaching worldwide a great number of users. Recently, zbMATH has created an account on Twitter. Here you can check on news about zbMATH, important mathematical news, information about mathematicians, history, and some anecdotes. You can find us at @zbMATH, or you can click at the twitter icon at the page footer of zbMATH.

\section{MSC2020: work in progress}

After the feedback of the mathematical community, the common effort between zbMATH and Mathematical Reviews is going forward to revise and improve the current Mathematics Subject Classification (MSC)
The joint MR-\#zbMATH reviewer reception at the \#ICM2018 continues a tradition which started alreay in 1998! MR Executive Editor Ed Dunne, EMS President Pavel Exner, AMS Executive Director Catherine Roberts, and zbMATH Editor-in-Chief Klaus Hulek address the reviewers.

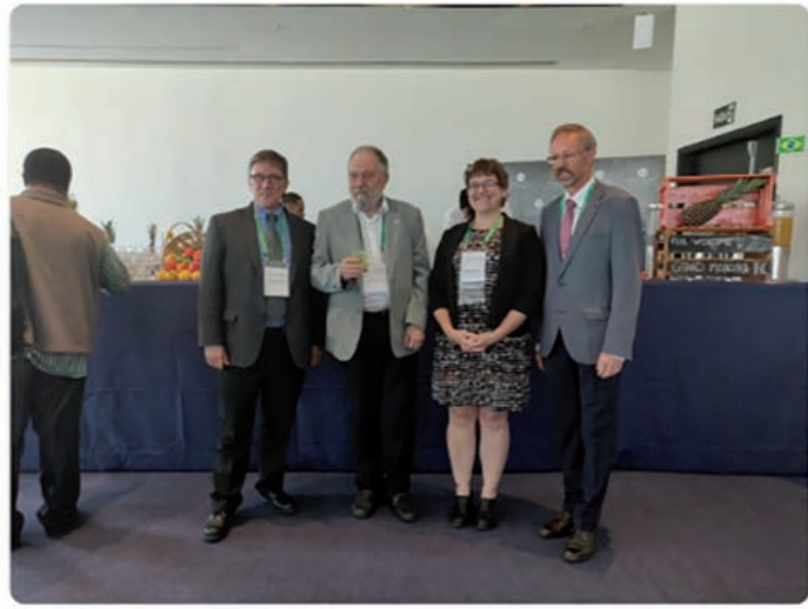

7:12 AM - 6 Aug 2018

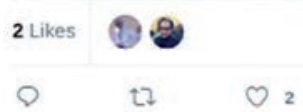

schema. Several concrete proposals and modifications are already being considered by both staffs. The results of this work will be announced at the end of this year.

Photo and CV of the author can be found in previous Newsletter issues.
European Mathematical Society Publishing House Seminar for Applied Mathematics

ETH-Zentrum SEW A21, CH-8092 Zürich, Switzerland subscriptions@ems-ph.org / www.ems-ph.org

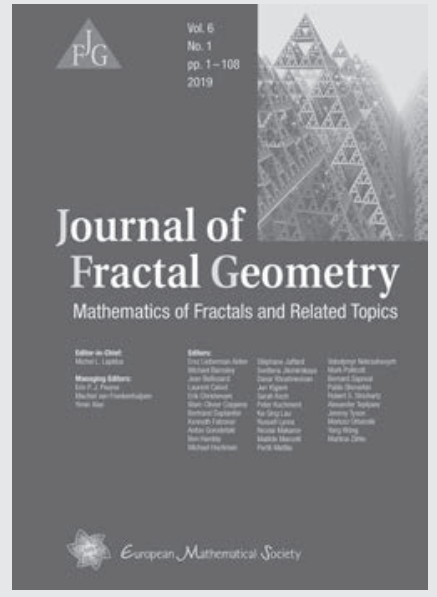

ISSN print 2308-1309

ISSN online 2308-1317

2019. Vol. 6.4 issues

Approx. 400 pages.

$17.0 \times 24.0 \mathrm{~cm}$

Price of subscription:

198 € online only /

238 e print+online
Editor-in-Chief:

Michel L. Lapidus (University of California, Riverside, USA)

\section{Managing Editors:}

Erin P. J. Pearse (California State Polytechnic University, San Luis Obispo, USA)

Machiel van Frankenhuijsen (Utah Valley University, Orem, USA) Yimin Xiao (Michigan State University, East Lansing, USA)

\section{Aims and Scope}

The Journal of Fractal Geometry is dedicated to publishing high quality contributions to fractal geometry and related subjects, or to mathematics in areas where fractal properties play an important role. The journal accepts submissions of original research articles and short communications, occasionally also research expository or survey articles, representing substantial advances in the field. 